\title{
Case Series: Emergency Point-of-care Ultrasound Identification of Pediatric Ventriculoperitoneal Shunt Malfunctions
}

\author{
Authors: \\ Deborah Shellshear BSc MBBS FRACP1,2,3 \\ Peter James Snelling BSc MBBS MPHTM FRACP CCPU $2,3,4$ \\ Adam $0^{\prime}$ Brien MBBS FACEM DDU ${ }^{1}$ \\ Michael Barrett MD MRCPI1,5,6,7
}

1. Emergency Department, Royal Children's Hospital, Melbourne, Victoria, Australia

2. Emergency Department, Lady Cilento Children's Hospital, Brisbane, Queensland, Australia

3. School of Medicine, The University of Queensland, Brisbane, Queensland, Australia

4. Department of Paediatrics, Greenslopes Private Hospital, Brisbane, Queensland, Australia

5. Murdoch Children's Research Institute, Royal Children's Hospital, Melbourne, Australia

6. Paediatric Emergency Research Unit, National Children's Research Centre, Dublin

7. Emergency Department, Our Lady's Children's Hospital, Dublin, Ireland

\section{Corresponding author:}

Dr Peter James Snelling

peter.j.snelling@gmail.com

Lady Cilento Children's Hospital

Emergency Department

Level 7 Directorate, 501 Stanley St

South Brisbane

Qld, Australia 4101

F: 61730684419

T: 61730688111

\section{Conflict of Interest}

Nil acknowledgements or disclosure of funding

\section{Authors' contributions}

DS drafted the initial manuscript with ongoing critical review. PJS revised the manuscript, contributed case 3 (including images) and submitted the final manuscript. AO critically reviewed the manuscript and contributed all other images. $\mathrm{MB}$ conceptualized the series and critically reviewed the manuscript. 


\section{Abstract:}

2 Ventriculoperitoneal shunt malfunctions should be accurately and efficiently diagnosed.

3 In this case series, we describe the use of point-of-care ultrasound to rapidly identify

4 pediatric ventriculoperitoneal shunt tubing fracture, obstruction and infection.

\section{CASE 1}

7 A 14 year-old male with a ventriculoperitoneal (VP) shunt was brought to the emergency 8 department (ED) with 3 days of headaches. The shunt was inserted 12 years prior for 9 hydrocephalus following pilocytic astrocytoma resection. Point-of-care ultrasound 10 (POCUS) of the VP shunt along the neck section of tubing revealed a fracture and a remnant calcified tract (Figure 1). The plain film shunt series affirmed the tubing fracture (Figure 2 online only) and computerized-tomography (CT) of the head excluded hydrocephalus. After consultation with neurosurgery, the fractured shunt tubing was 14 thought to be long-standing and non-contributory to the patient's current symptomatology. Therefore, neurosurgical intervention was not required and the patient was discharged home with outpatient follow-up.

POCUS findings (Figure 1): A longitudinal view of the VP shunt within the neck demonstrating a discontinuity of the proximal tubing, indicative of a fracture. The distal section of tubing had migrated caudally from view, leaving behind a patchy hyperechoic calcified tract with posterior acoustic shadowing.

\section{CASE 2}

24 A 4 month-old girl with a VP shunt inserted 3 weeks prior secondary to a pilocytic astrocytoma, was brought to the ED with 1 day of irritability. POCUS of the shunt was performed, with no scalp collection or tubing discontinuity identified. Cranial ultrasound (US) performed by the radiology department demonstrated stable ventricles without hydrocephalus and no CT was performed. The patient represented within a week with swelling over the shunt adjacent to the ear lobe and increased head circumference. POCUS identified an anechoic subcutaneous collection around the shunt skull insertion site (Figure 3). This was presumed to be cerebrospinal fluid (CSF) in the setting of shunt obstruction, as there were no signs of infection. The neurosurgical team was consulted and deemed it appropriate for inpatient brain magnetic resonance imaging (MRI), which 
confirmed VP shunt obstruction, with ventriculomegaly and a pseudomeningocele adjacent to the shunt insertion site. The patient was discharged after shunt revision.

POCUS findings (Figure 3): Longitudinal view of the VP shunt near the skull insertion site demonstrating an anechoic subcutaneous collection (CSF).

\section{CASE 3}

A 16 year-old male presented to the ED with increasing pain 3 weeks after a VP shunt revision with new tubing inserted. His original shunt was inserted as an infant for hydrocephalus following pilocytic astrocytoma resection, with the original tubing remaining in situ. There was warmth, erythema and tenderness across his right anterior chest wall overlying his new shunt tubing. The POCUS revealed a heterogeneous collection surrounding intact tubing in this region with adjacent echogenic inflammatory changes (Figure 4). Urgent neurosurgical review was sought for a presumptive diagnosis of shunt tubing infection. Shunt series and CT head excluded shunt discontinuity or obstruction. CSF tapped from the shunt remained culture negative but methicillinsensitive Staphylococcus aureus was cultured from the distal end of the tubing. He was commenced on intravenous antibiotic coverage and underwent externalization of the shunt. The infected tubing was removed with eventual re-internalization of a new VP shunt tubing and discharge home.

POCUS findings (Figure 4): Longitudinal (a) and transverse (b) views of the VP shunt tubing in the right anterior chest wall. A hypoechoic, heterogeneous collection immediately surrounding the tubing with adjacent hyperechoic subcutaneous tissue was demonstrated. The unaffected remnant original shunt tubing can be visualized in the transverse view.

\section{TECHNIQUE}

63 The components of a VP shunt include the proximal tubing, reservoir, valve and distal tubing. POCUS of a VP shunt is performed with a high-resolution linear array probe, with the marker either towards the patient's head (longitudinal) or right side (transverse). The patient is positioned comfortably in a supine or sitting position and the shunt is 
shunt until terminating at the distal tubing as it enters the abdomen. The shunt tubing is identified as a linear (longitudinal) or circular (transverse), non-compressible, fluidfilled structure with "tram-tracks" outlining the wall (Figure 5 online only). Limitations to sonographic evaluation of a VP shunt include include thick hair obscuring the view of the scalp components, the concave surface above and below the clavicle making positioning a linear transducer difficult, and bowel gas obscuring the view of the peritoneal tubing.

\section{REVIEW OF THE LITERATURE}

VP shunt complications are common, with $80 \%$ of pediatric patients having at least one episode of dysfunction within the first 10 years of insertion. ${ }^{1}$ Half of these episodes occur in the first year and $14 \%$ in the first month post insertion. ${ }^{2,3}$ VP shunt malfunction often results from tubing fracture (Case 1), which occurs commonly in the neck, ${ }^{2}$ obstruction (Case 2) or infection (Case 3). Other complications include peri-skull disconnection, valve failure, coiling and spontaneous knot formation in the catheter, and migration of the distal catheter into another body cavity. ${ }^{2-4}$

Typical radiological methods utilized to diagnose VP shunt dysfunction include plain-film shunt series and head CT. ${ }^{5}$ These patients often have a shunt series each time they present to the ED, amounting to a significant quantity of ionizing radiation exposure over a lifetime..$^{5-6}$ Subsequent investigation with CT results in additional high-dose ionizing radiation. ${ }^{7}$ However, the alternative option of rapid MRI in the ED remains poorly available. ${ }^{8}$

The utility of US in the assessment of VP shunt dysfunction has been described. ${ }^{9-11}$ Cranial US has become the imaging modality of choice in Australia for the initial diagnosis and monitoring of ventriculomegaly in neonates and infants with a patent anterior fontanelle. ${ }^{9}$ US has also been used to diagnose distal complications including catheter migration into the scrotum ${ }^{10}$ and in the identification of intra-abdominal CSF pseudocyst formation. ${ }^{11}$

POCUS can be rapidly performed to diagnose VP shunt complications and has the potential to obviate further imaging. It has been previously reported to diagnose shunt 
101 tubing fracture ${ }^{12}$, intra-abdominal CSF pseudocyst formation ${ }^{13}$ and infection. ${ }^{14}$ Along 102 with sparing ionizing radiation, other advantages of POCUS over shunt series include the 103 ability to image the radiolucent valve and reservoir and detect a subcutaneous collection, 104 which could indicate CSF leakage or infection. ${ }^{14}$

\section{CONCLUSIONS}

107 Our case series has demonstrated the use of POCUS in the evaluation of VP shunts. The 108 potential for early, rapid and accurate POCUS diagnosis of VP shunt dysfunction without 109 ionizing radiation makes this modality highly appealing to the ED physician. Further 110 research with a prospective study is required to evaluate this hypothesized use.

\section{Conflict of Interest}

113 None declared

\section{REFERENCES}

117 1. Bondurant CP JD. Epidemiology of Cerebrospinal Fluid Shunting. Pediatr 118 Neurosurg. 1995(23):254-9.

1192 2. Madikians A CE. Cerebrospinal fluid shunt problems in pediatric patients. Pediatr 120 Ann. 1997;26:613-20.

121 3. McGirt MJ LJ, Wellons JC, Villavicencio AT, Hopkins JS, Fuchs HE, George TM.

122 Cerebrospinal fluid shunt survival and eitiology of failures: a seven-year institutional 123 experience. Pediatr Neurosurg. 2002;36:248-55.

124 4. Boyle TP NL. Radiographic evaluation of Pediatric Cerebrospinal Fluid Shunt 125 Malfunction in the Emergency Setting. Pediatr Emerg Care. 2015;31:435-40.

126 5. Iskandar BJ MC, Mapstone TB, Grabb PA, Oakes WJ. Pitfalls in the diagnosis of 127 ventricular shunt dysfunction: radiology reports and ventricular size. Pediatrics. 128 1998;101:1031-6.

129 6. Desai KR BJ, Amosio JB. The utility of the plain radiograph "shunt series" in the 130 evaluation of suspected ventriculoperitoneal shunt failures in pediatric patients. Pediatr 131 Radiol. 2007;37:452-6.

132 7. Udavanskar UK BK, Arvaniti M, Tudorascu D, Small WC, Little S, Palasis S. Low133 dose nonenhanced head CT protocol for follow-up evaulation of children with 134 ventriculoperitoneal shunt: reduction of radiation and effect on image quality. $A m \mathrm{~J}$ 135 Neuroradiol. 2008;29:802-6.

136 8. Iskandar BJ SJ, Medow JM, Rowley HA. The use of quick-brain magnetic resonance 137 imaging in the evaluation of the shunt-treated hydrocephalus. J Neurosurg. 2004;101(2 138 suppl):147-51

139 9. RP G. Infant Craniospinal Ultrasonography: Beyond Hemorrhage and 140 Hydrocephalus. Semin Ultrasound CT MRI. 2010;31:71-95. 
141 10. Mohammadi A HA, Ghasemi-rad M. Scrotal migration of a ventriculoperitoneal 142 shunt: a case report and review of the literature. Medical Ultrasonography. 143 2012;14(2):158-60.

144 11. Briggs JR HG, Minns RA. Abdominal ultrasound diagnosis of cerebrospinal fluid 145 pseudocysts complicating ventriculoperitoneal shunts. Arch Dis Child. 1984;59:661-4.

146 12. Hamburg LM, Kessler DO. Rapid evaluation of ventriculoperitoneal shunt function 147 in a pediatric patient using emergency ultrasound. Pediatr Emer Care. 2012;28:726-727. 148 13. Ivan Y, Hauptman J, Marin JR. Abdominal cerebrospinal fluid pseudocyst diagnosed 149 by point-of-care ultrasound. Pediatr Emer Care. 2016;32:408-409.

150 14. Berg EA, Kashani S, Kang TL. More than just a abscess: ultrasound-assisted diagnosis 151 of ventriculoperitoneal shunt infection. West J Emerg Med. 2015;16(7):1180.

154 FIGURE 1. VP shunt tubing fracture depicted in its longitudinal plane. The distal end of 155 the shunt tubing is not visualized in the image due to caudal migration.

FIGURE 2. Chest radiograph as part of the shunt series demonstrating the fractured VP 158 shunt tubing.

FIGURE 3. Longitudinal view of the VP shunt demonstrating the anechoic subcutaneous CSF collection (pseudomeningocele).

163 FIGURE 4. Longitudinal (a) and transverse (b) views of the VP shunt tubing in the anterior chest wall, with a surrounding heterogeneous fluid collection and echogenic inflammatory changes.

166

FIGURE 5. Standard features of a VP shunt (INTEGRA® OSV). (a) Longitudinal comparison of actual and water bath ultrasound images. (b) Transverse view of tubing in situ.

170

\section{Authors' contributions}

173 DS drafted the initial manuscript with ongoing critical review. PJS revised the 174 manuscript, contributed case 3 (including images) and submitted the final manuscript. 175 AO critically reviewed the manuscript and contributed all other images. MB 176 conceptualized the series and critically reviewed the manuscript. 


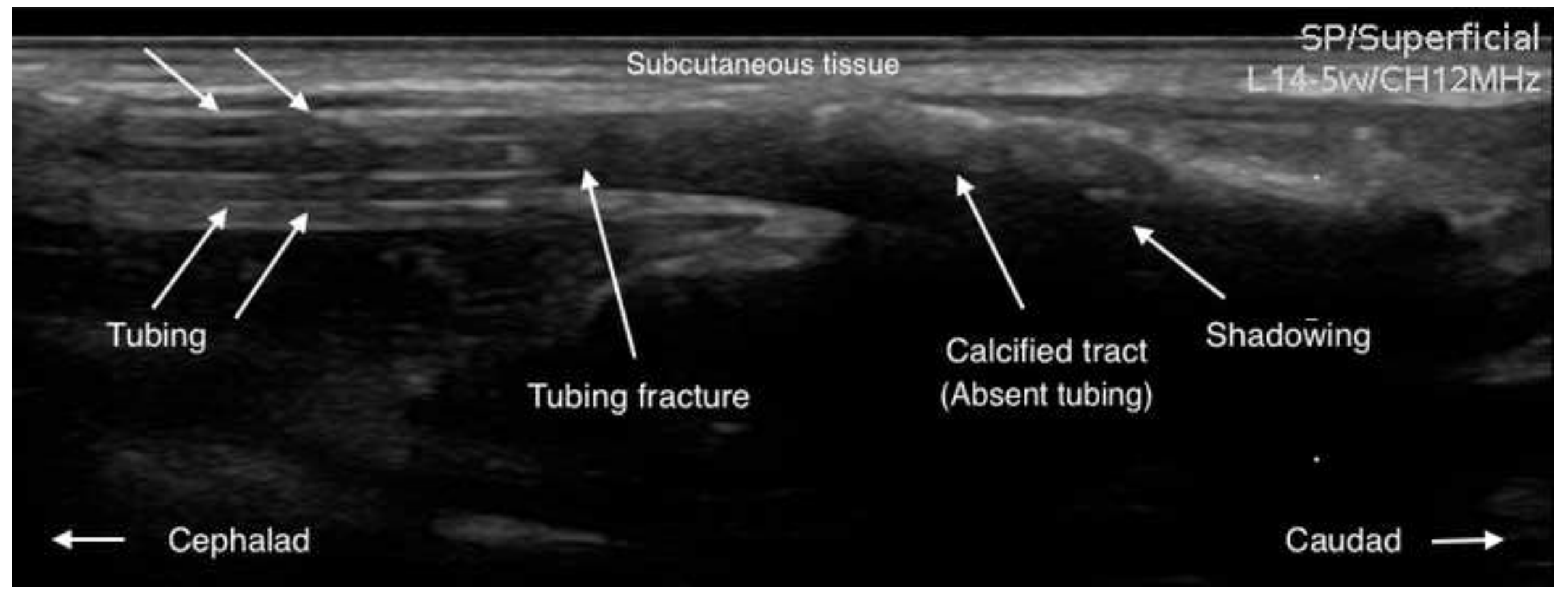




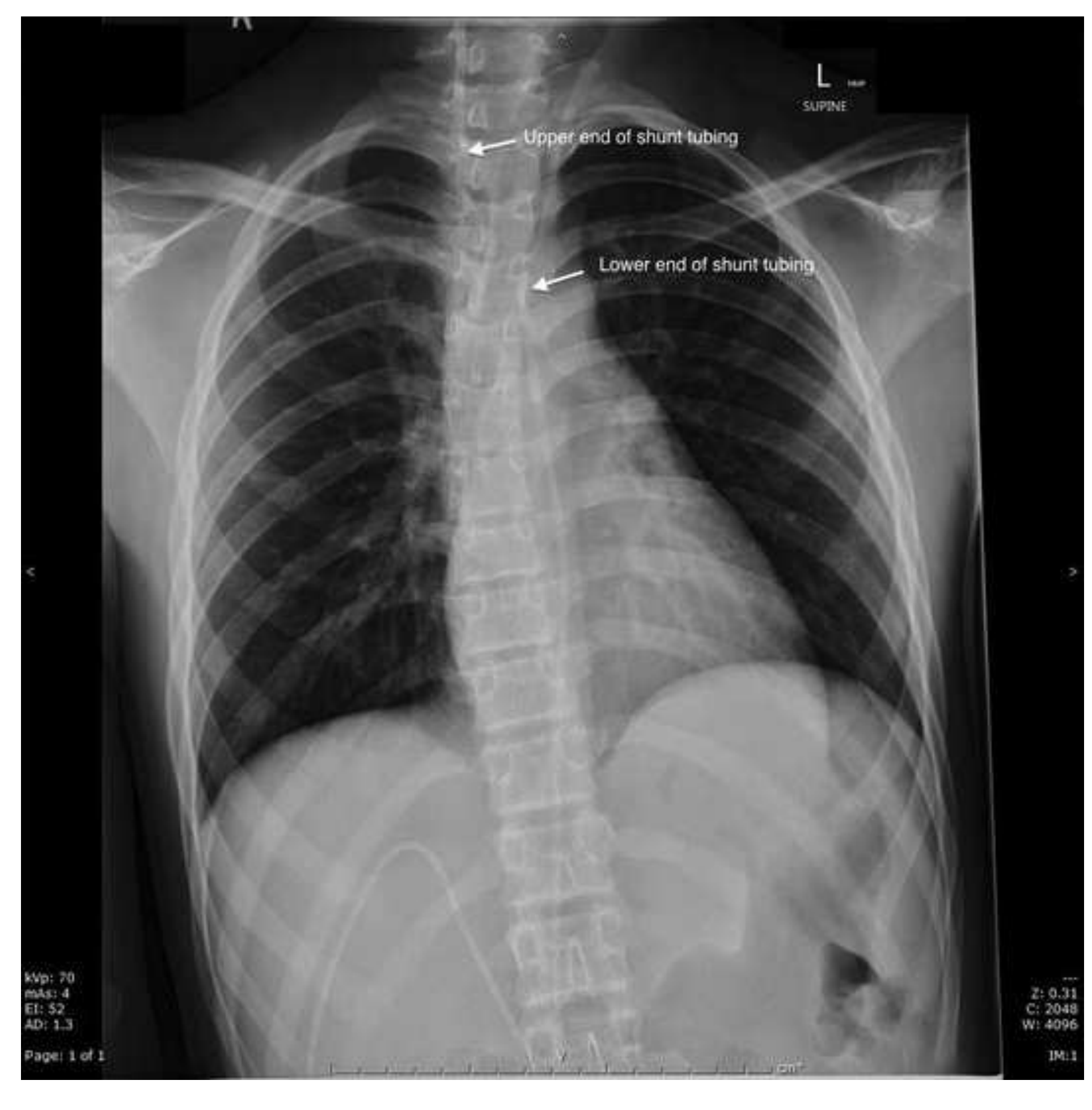

supks

1:5:

Paget 1 of 1

Figure 2

(1)

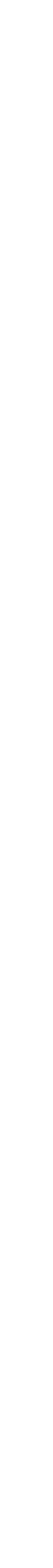




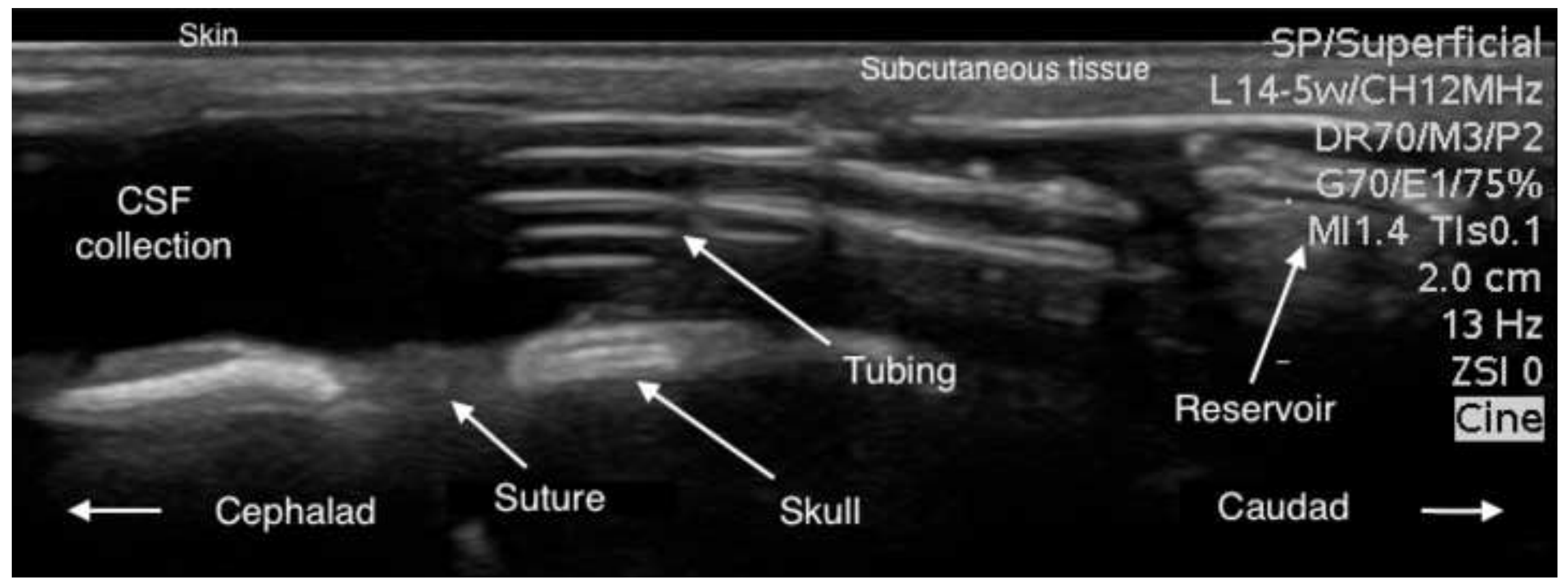




\section{Chest Wall}

Subcutaneous

Inflammatory changes

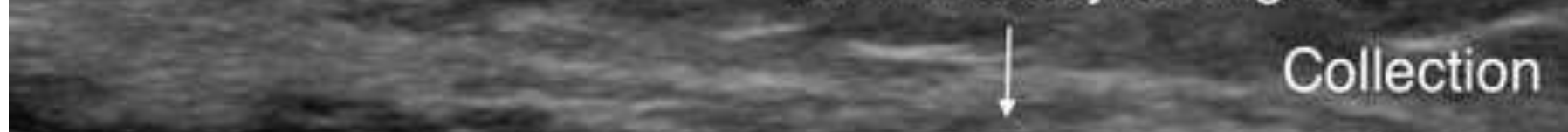

Shunt tubing
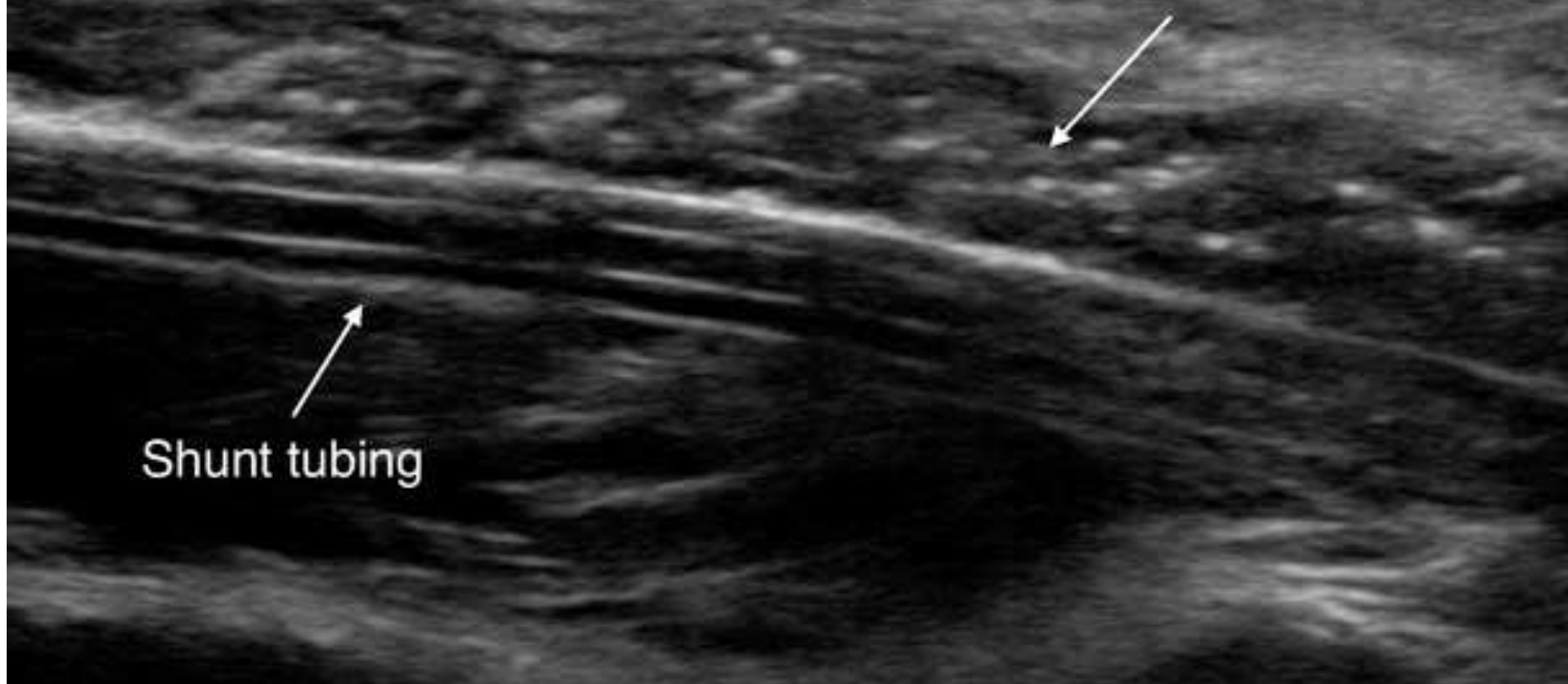

$\longleftarrow$ Cephalad

Caudad
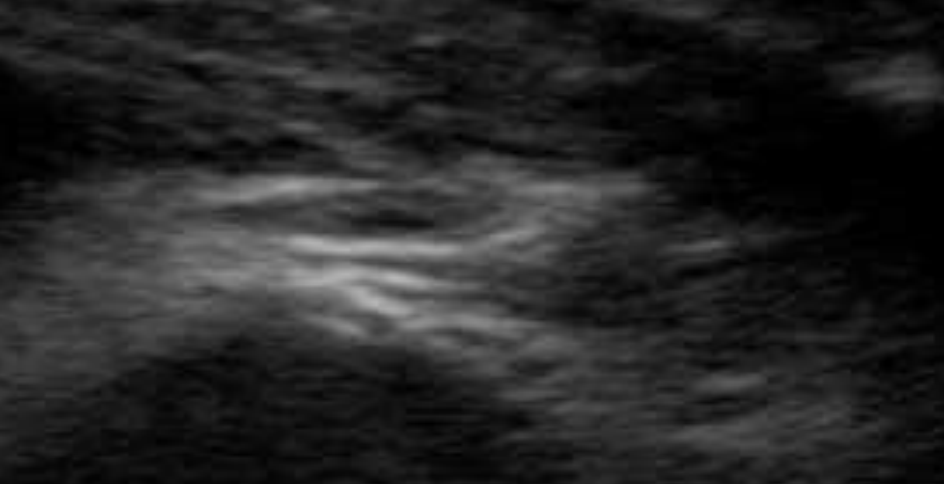

$$
\text { - }
$$




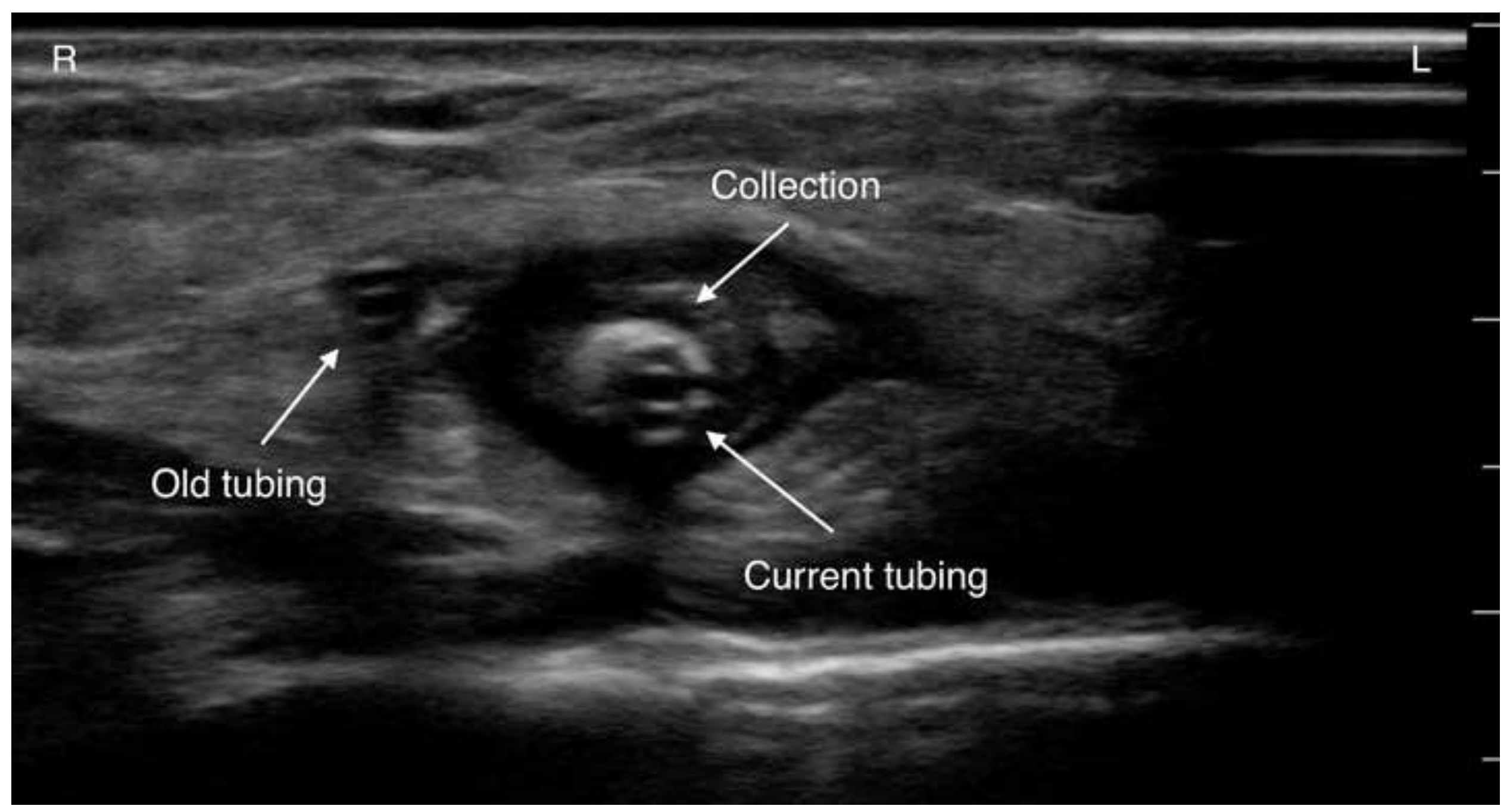




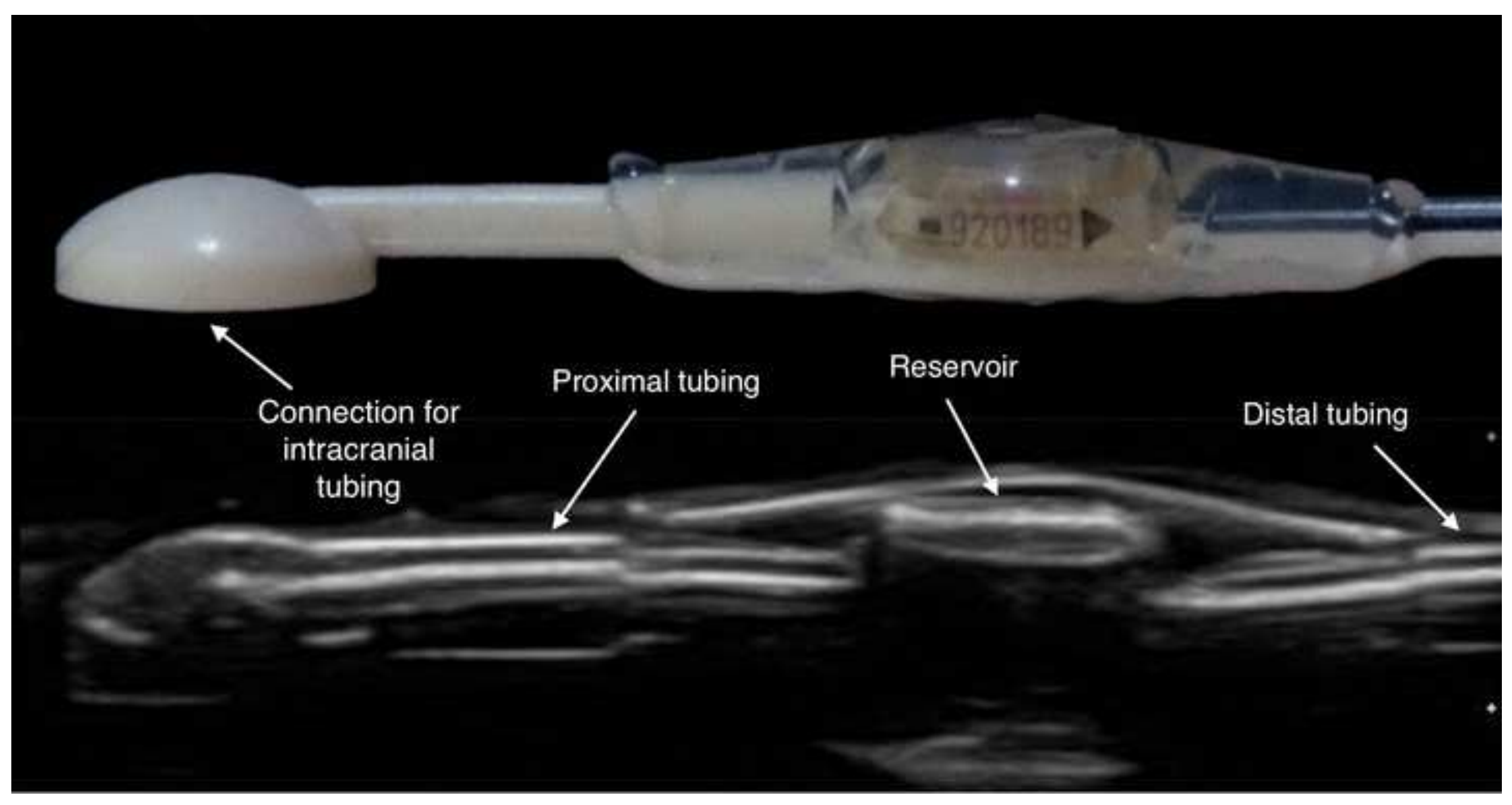


Click here to download Figure Figure 5b.jpg $\underline{\underline{\Perp}}$

\section{R}

\section{Transverse}

Anterior wail

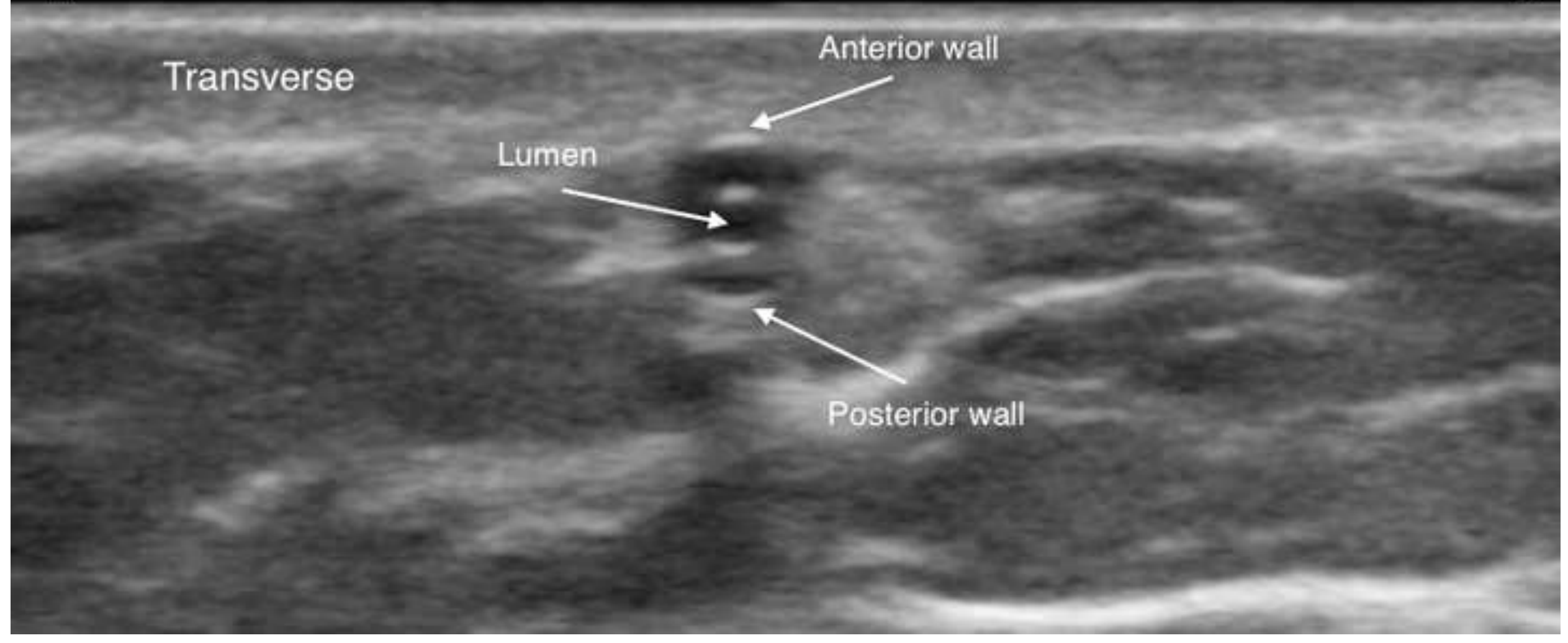

